\title{
Is treat-to-target really working in rheumatoid arthritis? a longitudinal analysis of a cohort of patients treated in daily practice (RA BIODAM)
}

\author{
Sofia Ramiro (1) 1,2 Robert BM Landewé, ${ }^{2,3}$ Désirée van der Heijde (1) , \\ Alexandre Sepriano $\odot 1,{ }^{1,4}$ Oliver FitzGerald, ${ }^{5}$ Mikkel Ostergaard, ${ }^{6}$ Joanne Homik, \\ Ori Elkayam, ${ }^{8}$ J Carter Thorne, ${ }^{9}$ Margaret Larche, ${ }^{10}$ Gianfranco Ferraciolli, ${ }^{11}$ \\ Marina Backhaus, ${ }^{12}$ Gilles Boire (1), ${ }^{13}$ Bernard Combe, ${ }^{14}$ Thierry Schaeverbeke, ${ }^{15}$ \\ Alain Saraux (1) ${ }^{16}$ Maxime Dougados, ${ }^{17}$ Maurizio Rossini (ㄷ , ${ }^{18}$ Marcello Govoni, ${ }^{19}$ \\ Luigi Sinigaglia, ${ }^{20}$ Alain G Cantagrel, ${ }_{1}^{21}$ Cornelia F Allaart, ${ }_{1}^{1}$ Cheryl Barnabe, ${ }_{1}^{22}$ \\ Clifton 0 Bingham (1) ${ }^{23}$ Paul P Tak (1) , ${ }^{3,24,25}$ Dirkjan van Schaardenburg, ${ }^{3}$ \\ Hilde Berner Hammer, ${ }_{1}^{26}$ Rana Dadashova, ${ }^{27}$ Edna Hutchings, ${ }_{1}^{27}$ Joel Paschke, \\ Walter P Maksymowych (1) 28
}

Handling editor Josef S
Smolen

For numbered affiliations see end of article.

\section{Correspondence to} Dr Sofia Ramiro, Rheumatology, Leiden University Medical Center, Leiden 2333 ZA, The Netherlands;

sofiaramiro@gmail.com

Received 12 December 2019 Revised 25 January 2020 Accepted 27 January 2020 Published Online First 24 February 2020

\section{Check for updates}

(C) Author(s) (or their employer(s)) 2020. No commercial re-use. See rights and permissions. Published by BMJ.

To cite: Ramiro S, Landewé RBM, van der Heijde D, et al. Ann Rheum Dis

2020:79:453-459.

\section{ABSTRACT}

Objectives To investigate whether following a treatto-target (T2T)-strategy in daily clinical practice leads to more patients with rheumatoid arthritis (RA) meeting the remission target.

Methods RA patients from 10 countries starting/ changing conventional synthetic or biological diseasemodifying anti-rheumatic drugs were assessed for disease activity every 3 months for 2 years (RA BIODAM (BIOmarkers of joint DAMage) cohort). Per visit was decided whether a patient was treated according to a T2T-strategy with 44-joint disease activity score (DAS44) remission (DAS44 $<1.6$ ) as the target. Sustained T2T was defined as T2T followed in $\geq 2$ consecutive visits. The main outcome was the achievement of DAS44 remission at the subsequent 3-month visit. Other outcomes were remission according to 28-joint disease activity scoreerythrocyte sedimentation rate (DAS28-ESR), Clinical Disease Activity Index (CDAI), Simplified Disease Activity Index (SDAI) and American College of Rheumatology/ European League Against Rheumatism (ACR/EULAR) Boolean definitions. The association between T2T and remission was tested in generalised estimating equations models.

Results In total 4356 visits of 571 patients (mean (SD) age: 56 (13) years, 78\% female) were included. Appropriate application of T2T was found in $59 \%$ of the visits. T2T (vs no T2T) did not yield a higher likelihood of DAS44 remission 3 months later (OR $(95 \% \mathrm{Cl})$ : 1.03 (0.92 to 1.16)), but sustained T2T resulted in an increased likelihood of achieving DAS44 remission (OR: 1.19 (1.03 to 1.39)). Similar results were seen with DAS28-ESR remission. For more stringent definitions (CDAl, SDAl and ACR/EULAR Boolean remission), T2T was consistently positively associated with remission (OR range: 1.16 to 1.29), and sustained T2T had a more pronounced effect on remission (OR range: 1.49 to 1.52). Conclusion In daily clinical practice, the correct application of a T2T-strategy (especially sustained T2T) in patients with RA leads to higher rates of remission.

\section{Key messages}

What is already known about this subject?

- Randomised controlled trials have demonstrated the efficacy of treat-to-target approaches in rheumatoid arthritis. Real life data from cohorts are still needed to support the widespread implementation of treat-totarget (T2T) in clinical practice.

What does this study add?

- In daily clinical practice, the correct application of a T2T-strategy in patients with rheumatoid arthritis (RA) leads to higher rates of remission as compared with not following it.

- Not only in early RA, but also in established RA, following a T2T-strategy leads to higher remission rates.

How might this impact on clinical practice or future developments?

- Rheumatologists should be encouraged to follow a T2T-strategy to contribute to the achievement of higher rates of remission for their patients.

\section{INTRODUCTION}

Early diagnosis, prompt commencement of disease modifying anti-rheumatic drug (DMARD) treatment and applying treat-to-target (T2T) strategies are now engrained in rheumatoid arthritis (RA) treatment paradigms. These approaches have substantially improved the outcomes of patients with RA. ${ }^{1}$ Remission has been defined and agreed on as the optimal target when managing a patient with RA. ${ }^{23}$ Reaching the state of remission is associated with reduced radiographic progression and improved functional ability. ${ }^{4}$

Thoroughly monitoring disease activity, adjusting treatment according to a fixed protocol and aiming 
at a predefined treatment goal, the so-called T2T-strategy, has advantages over usual care. ${ }^{5} 6$ Several strategy studies provide the basis of this evidence, namely the TICORA (Tight Control of RA study) ${ }^{7}$ and CAMERA (Computer Assisted Management in Early RA) ${ }^{8}$ studies. Subsequently, several strategy studies have incorporated a T2T-strategy in their treatment algorithm in the formal comparison of specific therapies, such as was done in the BeSt (Behandel Strategiëen) study. ${ }^{9}$ However, such evidence was gathered in the setting of randomised controlled trials (RCTs), with strict inclusion and exclusion criteria, following strict protocols and all particularities of RCTs. These studies provide the best evidence for the efficacy of $\mathrm{T} 2 \mathrm{~T}$ as an intervention, but to some extent compromise the generalisability of the findings, when one wants to consider applying them more broadly.

Having formally demonstrated the efficacy of T2T in RCTs, it is important to assess whether this strategy also improves outcomes in unselected patients from daily clinical practice. The first cohort studies focussed on patients with very early disease and confirmed that following a standardised intensive treatment led to improved achievement of remission. ${ }^{10}$ Subsequently, some cohort studies have shown that tight-control treatment leads to more rapid remission and higher remission achievement after 1 or 2 years than usual care. ${ }^{11} 12$ Nevertheless, the conclusions from these two studies were based on an indirect comparison between two different cohorts (one with T2T applied and another with usual care), with different patient characteristics, and focussed on the remission achievement at 1 or 2 years in the two cohorts. Such a comparison should ideally be made within the same cohort of patients, wherein some patients receive a T2T-strategy while others receive usual care. Real life data from cohorts without strict protocol specifications regarding choice of treatment are still needed to support the widespread implementation of T2T in clinical practice. Furthermore, previous studies have focussed on the achievement of remission at a given time point, for example, 1 or 2 years, ignoring whether or not the remission outcome was achieved in each of the visits throughout the follow-up (eg, three monthly visits, per T2T recommendations). A true longitudinal analysis taking all observations over time into account, both in terms of following T2T or not, and achieving remission or not, reflecting daily clinical practice, has not yet been conducted. Additionally, T2T has not yet been investigated in patients with established RA.

The aim of the present study was to investigate whether following a T2T-strategy leads to more patients with RA meeting the treatment target (remission) in daily clinical practice.

\section{METHODS}

\section{Study population}

Patients from RA BIODAM (BIOmarkers of joint DAMage), which has been previously described, were included. ${ }^{13}$ In brief, RA BIODAM is a 2-year multinational prospective observational study, including patients with a clinical diagnosis of RA and also fulfilling the 2010 RA Classification Criteria, ${ }^{14}$ recruited in daily practice from 10 countries from October 2011 to April 2015. To be eligible, patients presented with active disease (44-joint disease activity score, DAS44 $>2.4)^{15}$ and were to be started on or changing DMARD treatment, including conventional synthetic DMARDs (csDMARDs) and a first tumour necrosis factor inhibitor (TNFi); patients who had prior biological DMARD (bDMARD) experience were excluded. All patients were included in this analysis. The database used for this analysis was locked in April 2017. The study fulfilled Good Clinical Practice Guidelines and all patients provided informed consent.

\section{Remission}

Remission was the outcome of interest. According to the study protocol, patients were monitored every 3 months using DAS44 calculated with the erythrocyte sedimentation rate (ESR). ${ }^{15}$ DAS44 remission, that is, DAS44 $<1.6^{16}$ was therefore chosen as the main outcome for this analysis. Alternative definitions of remission were also used, namely the 28 -joint disease activity score $^{17}$ (DAS28-ESR) remission (ie, DAS28-ESR <2.6), ${ }^{18}$ the Clinical Disease Activity Index (CDAI) remission (ie, CDAI $\leq 2.8),{ }^{19}$ the Simplified Disease Activity Index (SDAI) remission $(\mathrm{SDAI} \leq 3.3)^{20}$ and the American College of Rheumatology/ European League Against Rheumatism (ACR/EULAR) Boolean remission (ie, tender joint count (TJC) $\leq 1$, swollen joint count $(\mathrm{SJC}) \leq 1$, C-reactive protein $(\mathrm{CRP}) \leq 1 \mathrm{mg} / \mathrm{dL}$ and patient global assessment (PGA) $(0$ to 10$) \leq 1) .^{2}$ All definitions of remission were binary (yes/no).

\section{Treat-to-target}

Participating rheumatologists were required by protocol to follow a T2T-strategy with DAS44 remission (DAS44<1.6) as benchmark. In order to define whether a T2T-strategy was appropriately followed or not, every visit was checked according to predefined criteria. T2T was considered appropriate: (i) if a patient had already a disease activity score below the target (DAS $<1.6$ ) and treatment was not intensified; or (ii) if treatment was intensified on a DAS $\geq 1.6$. Treatment intensification was defined as increasing dosage or adding a drug from the following categories: csDMARDs, bDMARDs or corticosteroids. T2T was considered incorrectly applied if: (i) the target was met and treatment was nevertheless intensified; or (ii) the target was not met and treatment was not intensified.

Additional definitions for T2T were also considered for sensitivity analyses: (i) T2T without corticosteroids, that is, without considering corticosteroids as a treatment intensification; (ii) T2T less strict, that is, considering T2T as adequate as long as the target, DAS44 remission, is met, regardless of whether treatment is nevertheless intensified or not; (iii) T2T-low disease activity (T2T-LDA) using LDA (ie, DAS $<2.4)^{21}$ instead of remission as the benchmark.

Furthermore, 'sustained T2T' strategy was defined as following $\mathrm{T} 2 \mathrm{~T}$ in at least two consecutive visits.

\section{Other relevant clinical information}

Age, gender, disease duration, rheumatoid factor (RF) and anticitrullinated protein antibodies (ACPA) status (positive/negative) and being DMARD-naïve (yes vs no), all collected at baseline, were considered in this analysis as potential effect modifiers or confounders of the relationship of interest. Country of residence was also considered as a potential confounder.

\section{Statistical analysis}

The relationship between following $\mathrm{T} 2 \mathrm{~T}$ at a given visit and meeting the target of remission at the subsequent visit 3 months later was investigated using time-lagged generalised estimating equations (GEE) models. GEE is a suitable technique to make use of all available observations from each patient while adjusting for inherent within-subject correlations of the repeated measurements. Models were time-lagged to allow investigation of the effect of the main predictor of interest (ie, following T2T) on the outcome (ie, remission) with a lag of 3 months; in other words, with the outcome occurring 3 months later. The same analyses were conducted to investigate the effect of sustained T2T on meeting the target of remission. The 'exchangeable' working correlation structure, demonstrating the best fit to the data, was used. 


\begin{tabular}{|c|c|}
\hline & $\begin{array}{l}n=571 \\
\text { mean (SD) or } n(\%)\end{array}$ \\
\hline Age, years & $55.7(12.9)$ \\
\hline Female gender & $434(76.0 \%)$ \\
\hline Disease duration, years & $6.5(8.0)$ \\
\hline Education, years & $12.6(3.8)$ \\
\hline Number of comorbidities & $1.2(1.3)$ \\
\hline Rheumatoid factor positivity & $370(68.0 \%)$ \\
\hline Anti-CCP positivity & $388(69.3 \%)$ \\
\hline RF and/or anti-CCP positivity & $431(77.7 \%)$ \\
\hline DAS44 (0-10) & $3.8(1.0)$ \\
\hline DAS28-ESR (0-10) & $5.2(1.2)$ \\
\hline CDAI (0-76) & $26.9(11.6)$ \\
\hline SDAI (0-86) & $28.5(12.4)$ \\
\hline Patient global (0-10) & $5.7(2.3)$ \\
\hline HAQ (0-3) & $1.1(0.7)$ \\
\hline SJC (0-44) & $8.4(6.1)$ \\
\hline TJC (0-53) & $13.6(9.1)$ \\
\hline $\mathrm{ESR}(\mathrm{mm} / \mathrm{h})$ & $28.7(22.2)$ \\
\hline CRP (mg/dL) & $1.5(2.3)$ \\
\hline Number of prior DMARDs & $0.9(1.1)$ \\
\hline DMARD naïve & $274(48.0 \%)$ \\
\hline \multicolumn{2}{|l|}{ Smoking status } \\
\hline Never smoker & $282(49.4 \%)$ \\
\hline Current smoker & $161(28.2 \%)$ \\
\hline Ex-smoker & $128(22.4 \%)$ \\
\hline \multicolumn{2}{|l|}{$\begin{array}{l}\text { Treatment csDMARD/TNFi started at } \\
\text { baseline }\end{array}$} \\
\hline Both & $196(34.6 \%)$ \\
\hline csDMARD only & $334(58.9 \%)$ \\
\hline TNFi only & $36(6.3 \%)$ \\
\hline None & $1(0.2 \%)$ \\
\hline $\begin{array}{l}\text { Treatment with oral corticosteroids } \\
\text { started at baseline }\end{array}$ & $255(45 \%)$ \\
\hline \multicolumn{2}{|c|}{$\begin{array}{l}\text { anti-CCP, anti-citrullinated protein; CDAI, Clinical Disease Activity Index; CRP, } \\
\text { C-reactive protein; cSDMARD, conventional synthetic DMARD; DAS44, 44-joint } \\
\text { disease activity score; DAS28-ESR, 28-joint disease activity score (with ESR); } \\
\text { DMARD, disease modifying anti-rheumatic drug; ESR, erythrocyte sedimentation } \\
\text { rate; HAQ, Health Assessment Questionnaire; RF, rheumatoid factor; SDAI, Simple } \\
\text { Disease Activity Index; SJC, swollen joint count; TJC, tender joint count; TNFi, tumour } \\
\text { necrosis factor inhibitor. }\end{array}$} \\
\hline
\end{tabular}

As treatment intensification has a central role in $\mathrm{T} 2 \mathrm{~T}$, we sought to investigate the extent to which the components of the disease activity scores contributed to it. We therefore investigated the effect of TJC $>1$, SJC $>1$, PGA $>1$ and CRP $>1 \mathrm{mg} /$ $\mathrm{dL}$ on treatment intensification (yes/no). This analysis was also conducted with GEE, including all above-mentioned disease activity components in one multivariable model.

For each model, interactions between the T2T variable and age, gender, disease duration and RF/ACPA positivity were tested, and if significant $(p<0.15)$ and clinically relevant the model was fitted in each subgroup. If these proved to be not relevant, final models were adjusted for potential confounders selected a priori: age, gender, disease duration and country of residence. Stata/SE V.12 was used.

\section{RESULTS}

In total, 571 patients were included with a mean age of 56 (SD 13) years, $78 \%$ females and a mean disease duration of 6.5 (8.0) years, $37 \%$ with a disease duration up to 2 years (table 1 ). In total, $78 \%$ of the patients were RF and/or ACPA positive, and $48 \%$ were DMARD-naive at baseline (mean disease duration of 3.6 (5.6), 50\% with $\leq 2$ year disease duration). At the end of the baseline visit, almost $60 \%$ of the patients were on treatment with csDMARDs only, $35 \%$ of the patients on a TNFi with a csDMARD and only $6 \%$ on TNFi monotherapy. Almost half of the patients were on corticosteroids after the baseline visit.

T2T was appropriately applied in 59\% of 4356 visits. This included $31 \%$ of patient visits where DAS44 remission was met and treatment was not intensified, and $28 \%$ of visits where treatment was appropriately escalated. In 3\% of visits $(9 \%$ of those with treatment intensification), treatment intensification took place even though DAS44 remission was met (making a total of $31 \%$ of the visits with treatment intensification). In the remaining $38 \%$ of visits T2T was not being followed as there was no treatment intensification despite active disease (DAS44 $\geq 1.6$ ) (figure 1 ).

Throughout the 2-year follow-up period an increasing proportion of patients met remission definitions. At 3 months $24 \%$ of the patients were in DAS44 and DAS28-ESR remission, and 8\% in ACR/EULAR Boolean remission. At 24 months 52\% of the patients were in DAS44 remission, also 52\% in DAS28-ESR remission and 27\% in ACR/EULAR Boolean remission (figure 2).

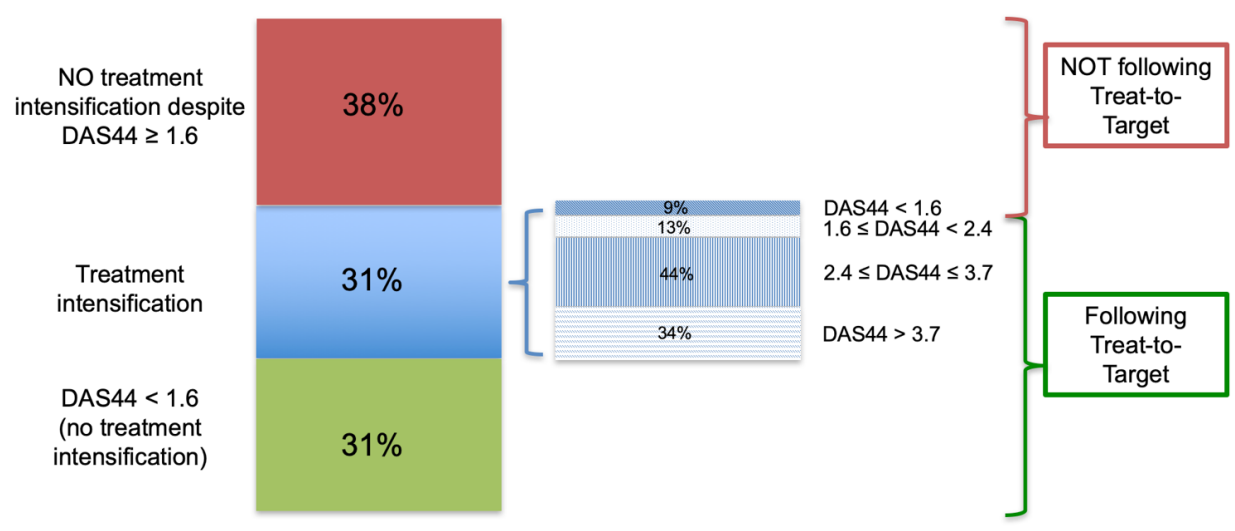

Figure 1 Proportion of the visits ( $n=4356$ ) in which treat-to-target strategy (with DAS44 $<1.6$ as benchmark) is followed versus not and the details regarding the proportion of visits with target achievement and/or treatment intensification. Treatment intensification was defined as start or dosage increase of a conventional synthetic or biological disease modifying anti-rheumatic drug or of a corticosteroid. DAS44: 44-joint disease activity score. 


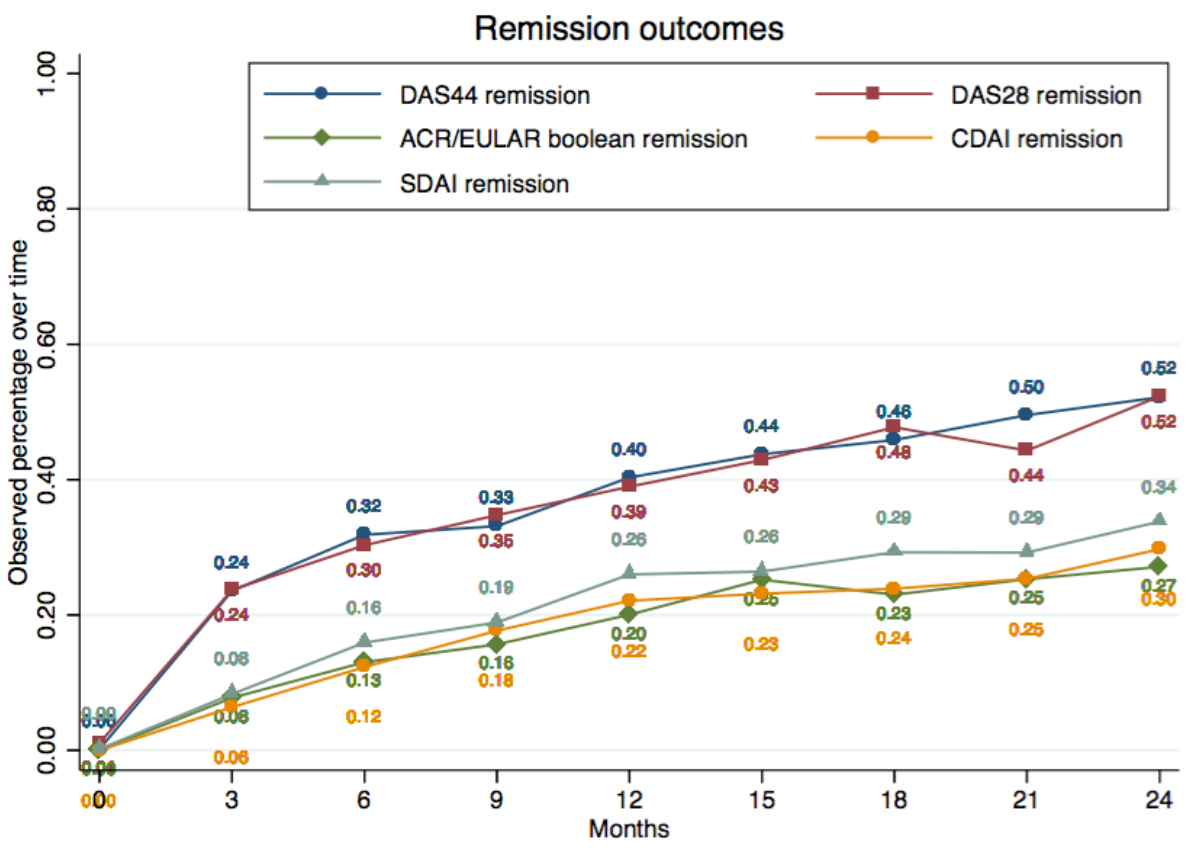

Figure 2 Proportion of achievement of the different remission outcomes throughout the 2-year follow-up. ACR,American College of Rheumatology; CDAI, ClinicalDisease Activity Index; DAS28, 28-joint disease activity score; DAS44, 44-jointdisease activity score; EULAR, European League Against Rheumatism; Simplified Disease Activity Index (SDAI).

\section{T2T on remission outcomes}

Following a T2T-strategy, as compared with not following it, was not significantly associated with a DAS44 or DAS28-ESR remission 3 months later (OR (95\% CI) 1.03 (0.92 to 1.16) and 1.03 (0.91 to 1.16), respectively), but was significantly associated with ACR/EULAR Boolean remission (OR 1.16 (1.01 to 1.34)) and also with CDAI remission (OR 1.29 (1.12 to 1.49)) and SDAI remission (OR 1.24 (1.08 to 1.41)) (table 2). Results of the sensitivity analyses were similar, except for a slightly stronger association between T2T and remission (REM) outcomes for both 'T2T without corticosteroids' and 'T2T-REM less strict'. With T2T-LDA, with LDA as the benchmark, there was a significant association between T2T and all remission outcomes (OR between 1.3 and 1.4). None of the tested effect modifiers, namely age, gender, disease duration, seropositivity or DMARD naïve (vs not), modified the relationships of interest.

\section{Sustained T2T on remission outcomes}

Following a sustained T2T-strategy compared with not following it was associated with remission 3 months later according to all definitions, for example, DAS44 remission OR 1.19 (1.03 to 1.39 ) or ACR/EULAR Boolean remission (OR 1.49 (1.24 to 1.81)) (table 3).

\section{Relationship between disease activity components and treatment intensification}

All disease activity components were significantly associated with treatment intensification, with SJC and TJC showing the strongest associations, also in a multivariable model including all the components: OR 'SJC >1' 3.42 (2.89 to 4.05), OR 'TJC >1' 3.35 (2.72 to 4.11$)$, OR 'PGA >1' 2.14 (1.71 to 2.68$)$ and $\mathrm{OR}$ 'CRP > 1' 2.00 (1.66 to 2.42).

\begin{tabular}{|c|c|c|c|c|c|}
\hline & $\begin{array}{l}\text { DAS44 remission } \\
(0 \mathrm{OR}(95 \% \mathrm{CI}))\end{array}$ & $\begin{array}{l}\text { DAS28-ESR remission } \\
(\mathrm{OR}(95 \% \mathrm{CI}))\end{array}$ & $\begin{array}{l}\text { ACR/EULAR Boolean remission } \\
(\mathrm{OR}(95 \% \mathrm{Cl}))\end{array}$ & $\begin{array}{l}\text { CDAI remission } \\
(\text { OR }(95 \% \mathrm{CI}))\end{array}$ & $\begin{array}{l}\text { SDAI remission } \\
(\mathrm{OR}(95 \% \mathrm{CI}))\end{array}$ \\
\hline $\mathrm{T} 2 \mathrm{~T}$ & $1.03(0.92$ to 1.16$)$ & 1.03 (0.91 to 1.16$)$ & 1.16 (1.01 to 1.34$)$ & 1.29 (1.12 to 1.49$)$ & $\begin{array}{l}1.24(1.08 \text { to } \\
1.41)\end{array}$ \\
\hline T2T without corticosteroids & $1.07(0.95$ to 1.20$)$ & 1.11 (0.98 to 1.26$)$ & 1.23 (1.06 to 1.42 ) & 1.37 (1.18 to 1.59$)$ & $\begin{array}{l}1.34(1.17 \text { to } \\
1.53)\end{array}$ \\
\hline T2T-REM less strict & 1.06 (0.94 to 1.19$)$ & 1.07 (0.95 to 1.21$)$ & $1.32(1.13$ to 1.53$)$ & $1.43(1.22$ to 1.67$)$ & $\begin{array}{l}1.34(1.17 \text { to } \\
1.54)\end{array}$ \\
\hline T2T-LDA & 1.26 (1.10 to 1.44$)$ & $1.36(1.17$ to 1.56$)$ & 1.27 (1.09 to 1.47$)$ & 1.39 (1.18 to 1.64$)$ & $\begin{array}{l}1.36(1.17 \text { to } \\
1.59)\end{array}$ \\
\hline
\end{tabular}

T2T without corticosteroids: without considering corticosteroids in treatment intensification. T2T-REM less strict: considering T2T as adequate as long as the target, DAS44 remission, is met, regardless of whether treatment nevertheless intensified or not.

*All models adjusted for age, gender, disease duration and country. T2T was considered being followed: (i) if a patient had already a disease activity score below the target (DAS $<1.6$; DAS $<2.4$ for LDA definition) and treatment was correctly not intensified; or (ii) if treatment was intensified on a DAS $\geq 1.6$ (or DAS $\geq 2.4$ for LDA definition). ACR, American College of Rheumatology; CDAl, Clinical Disease Activity Index; DAS44, 44-joint disease activity score; DAS28-ESR, 28-joint disease activity score (with ESR); ESR, erythrocyte sedimentation rate; EULAR, European League Against Rheumatism; SDAI, Simple Disease Activity Index; T2T, treat-to-target; T2T-LDA, T2T-low disease activity; T2TREM, T2T-remission. 
Table 3 Effect of following a sustained treat-to-target strategy on remission outcomes 3 months later*

\begin{tabular}{llllll}
\hline & $\begin{array}{l}\text { DAS44 sustained } \\
\text { remission } \\
(\mathrm{OR}(95 \% \mathrm{Cl}))\end{array}$ & $\begin{array}{l}\text { DAS28-ESR sustained } \\
\text { remission } \\
(\mathrm{OR}(95 \% \mathrm{CI}))\end{array}$ & $\begin{array}{l}\text { ACR/EULAR Boolean } \\
\text { sustained remission } \\
\text { (OR }(95 \% \mathrm{Cl}))\end{array}$ & $\begin{array}{l}\text { CDAI sustained remission } \\
\text { (OR }(95 \% \mathrm{Cl}))\end{array}$ & $\begin{array}{l}\text { SDAl sustained remission } \\
\text { (OR }(95 \% \mathrm{Cl}))\end{array}$ \\
\hline Sustained T2T & $1.19(1.03$ to 1.39$)$ & $1.23(1.06$ to 1.44$)$ & $1.49(1.24$ to 1.81$)$ & $1.45(1.19$ to 1.77$)$ & $1.52(1.27$ to 1.82$)$ \\
\hline
\end{tabular}

*All models adjusted for age, gender, disease duration and country. Sustained treat-to-target was considered followed if T2T was followed in $\geq 2$ subsequent visits. T2T was considered being followed: (i) if a patient had already a disease activity score below the target (DAS $<1.6$; DAS $<2.4$ for LDA definition) and treatment was correctly not intensified; or (ii) if treatment was intensified on a DAS $\geq 1.6$ (or DAS $\geq 2.4$ for LDA definition).

ACR, American College of Rheumatology; CDAl, Clinical Disease Activity Index; DAS44, 44-joint disease activity score; DAS28-ESR, 28-joint disease activity score; ESR, erythrocyte sedimentation rate; EULAR, European League Against Rheumatism; LDA, low disease activity; SDAI, Simple Disease Activity Index; T2T, treat-to-target.

\section{DISCUSSION}

In the present study we have shown that following a T2Tstrategy, and particularly sustained T2T, in daily clinical practice leads to more patients with RA meeting the most stringent remission criteria over time. This is the first comprehensive analysis that considers all available visits of unselected patients who were followed by protocol for a period of 2 years. The results of the analysis provide direct evidence that following $\mathrm{T} 2 \mathrm{~T}$, and particularly sustained $\mathrm{T} 2 \mathrm{~T}$, immediately results in a higher likelihood of remission at the next visit, 3 months later (the longitudinal interpretation of a T2T-strategy). Moreover, we have for the first time shown that following T2T is also efficacious in patients with established RA, while previous studies focussed on the effect of T2T in patients with early RA.

The strictly temporal relationship between following a T2T-strategy and meeting remission was statistically significant for almost all remission outcomes and for the different T2T definitions used. The exceptions were the DAS44 and DAS28-ESR remission definitions with an interval of 3 months only, while for sustained T2T the relationship with all remission outcomes was statistically significant. The explanation is rather technical: the independent variable (T2T with DAS44 as benchmark) and the outcome (ie, DAS44 remission) include exactly the same disease activity score, which implies that the model becomes inherently auto-regressive. Such a scenario effectively removes the variability in the data necessary to demonstrate efficacy of an intervention. The other definitions of remission are slightly different from the benchmark definition and allow more statistical separation. An alternative explanation is that DAS44 and DAS28-ESR definitions are more lenient in comparison to ACR/EULAR Boolean, CDAI and SDAI remission and are more frequently met even if T2T is not applied. ${ }^{2}$ Nevertheless, the signal that a T2T-strategy, and particularly sustained T2T-strategy, increases the likelihood of stringent remission is clear and consistent. Also, these findings became even more evident throughout the follow-up of this study. The proportion of patients achieving remission, regardless of its definition, increased substantially through follow-up (figure 2). Even after 2 years, a plateau has not yet been reached, reassuring clinicians that if we measure disease activity and treat patients effectively over time, high remission rates can be achieved.

These findings come from a population of patients with an average of 6.5 years of disease duration. One may speculate that the effect of following T2T could be even better in early disease. In this study, we have not found any differences between patients DMARD naïve versus not and also according to disease duration, but a lack of statistical power cannot be excluded. Additionally, even patients who were DMARD naïve had a relatively long disease duration (average of 3.6 years), not being the most representative DMARD naïve patients.
If $\mathrm{T} 2 \mathrm{~T}$ is so clearly associated with clinical remission, as shown here and in the literature, ${ }^{56}$ why, then, is a T2T-strategy not always followed in clinical practice? Even in this study, with a protocol requiring implementation of T2T, this strategy was 'only' followed in less than two-thirds of the visits. Also within the RA BIODAM cohort, we have shown that, among other factors, the absence of objective signs of inflammation (eg, swollen joints) implied a lower likelihood to follow T2T. ${ }^{22}$ Also, in the 10 -year follow-up of the BeSt trial, nonadherence to the protocol has been assigned to disagreement with how DAS reflects disease activity (felt to overestimate the real disease activity) and disagreement with the subsequently required step in the protocol. ${ }^{23}$ Many clinicians find regularly measuring disease activity too time consuming endeavour and consider it an additional barrier to implementation of T2T. ${ }^{24} 25$

In order to launch new strategies or interventions in clinical practice, the formulation of recommendations, like the T2T recommendations, ${ }^{26}$ does not suffice and implementation should actively be promoted. Studies like ours may further corroborate the message that $\mathrm{T} 2 \mathrm{~T}$ leads to more stringent remission and may help implementation in clinical practice. Appropriate education may also help. The intervention of the TRACTION trial included one educational face-to-face meeting and monthly webinars on the principles and practical advice on implementation of T2T. A substantial improvement in the adherence to T2T was demonstrated with improvement of $46 \%$ in the arm following the training programme compared with $14 \%$ in the control arm. ${ }^{27}$ Still, rheumatologists may report compliance with recommendations but in practice do not always follow them. ${ }^{28}$

Some limitations of this study need to be considered. First, it is designed as an observational study reflecting daily clinical practice with unselected patients contrasting with the reality of RCTs from which most evidence on T2T originates to date. However, one may question how close to daily clinical practice the RA BIODAM cohort really is, with participation from only a few centres per country, several being tertiary referral centres, and with rheumatologists mandated to follow a strict T2T protocol. As in principle, rheumatologists were required to follow T2 T per protocol, we have in this study in essence compared the visits in which the protocol was followed to others in which protocol was violated. One can therefore not exclude a bias intrinsic to this comparison. Additionally, detailed reasons for not following T2T have not been adequately registered precluding additional analysis of adherence to T2T versus taking the physician's reasoning into account. Moreover, only patients with active disease were included, and the average baseline disease activity was high. This may preclude the generalisability of the findings to patients with low disease activity, and not answer the question of whether following a T2T-strategy is beneficial in patients 
already in low disease activity, given the risks of overtreatment. ${ }^{29}{ }^{30}$ Lastly, when investigating the impact of following a T2T-strategy, one is not only analysing the impact of treatment intensification but implicitly one is evaluating visits in which patients are already in remission, which have accentuated the benefit of T2T. However, it was our aim to investigate the impact of following the T2T-strategy in its whole and not parts of it, as well as to take all disease activity measurements into account as the longitudinal technique chosen properly does. As a main strength, this is a multinational observational study, including unselected patients reflecting daily clinical practice, with the first truly longitudinal analysis addressing the impact of following a (sustained) T2T-strategy.

In conclusion, following a T2T-strategy, and especially sustained T2T, works in daily clinical practice and leads to more patients meeting the target, that is, remission. Rheumatologists should be encouraged to follow a T2T-strategy to contribute to the achievement of higher rates of remission for their patients.

\section{Author affiliations}

'Department of Rheumatology, Leiden University Medical Center, Leiden, The Netherlands

${ }^{2}$ Department of Rheumatology, Zuyderland Medical Center, Heerlen, The Netherlands ${ }^{3}$ Department of Rheumatology, Amsterdam Rheumatology Center, Amsterdam, The Netherlands

${ }^{4}$ NOVA Medical School, Universidade Nova de Lisboa, Lisbon, Portugal

${ }^{5}$ St Vincent's University Hospital and Conway Institute for Biomolecular Research, University College Dublin, Dublin, Ireland

${ }^{6}$ Copenhagen Center for Arthritis Research, Center for Rheumatology and Spine Diseases, Rigshospitalet, Glostrup and Department of Clinical Medicine, University of Copenhagen, Copenhagen, Denmark

${ }^{7}$ Department of Medicine, University of Alberta, Edmonton, Alberta, Canada

${ }^{8}$ Tel Aviv Sourasky Medical Center and the "Sackler" Faculty of Medicine, Tel Aviv University, Tel Aviv, Israel

${ }^{9}$ Southlake Regional Health Centre, University of Toronto, Toronto, Ontario, Canada ${ }^{10}$ Departments of Medicine and Pediatrics, Divisions of Rheumatology, Clinical Immunolgoy and Allergy, McMaster University, Hamilton, Ontario, Canada

${ }^{11}$ Catholic University of the Sacred Heart, Roma, Italy

${ }^{12}$ Park-Klinik Weissensee, Academic Hospital of the Charité, Berlin, Germany

${ }^{13}$ Department of Medicine/Division of Rheumatology, Centre intégré universitaire de santé et de services sociaux de l'Estrie - Centre hospitalier universitaire de Sherbrooke (CIUSSS de I'Estrie-CHUS), Universite de Sherbrooke, Sherbrooke, Quebec, Canada

${ }^{14} \mathrm{CHU}$ Montpellier and Montpellier University, Montpellier, France

${ }^{15}$ Department of Rheumatology, FHU ACRONIM, University Hospital of Bordeaux, University of Bordeaux, Bordeaux, France

${ }^{16}$ Rheumatology, CHU Brest, Brest, France

${ }^{17}$ Rheumatology Department, Paris Descartes University, Cochin Hospital, Assistance Publique-Hôpitaux de Paris, INSERM (U1153): Clinical Epidemiology and Biostatistics, PRES Sorbonne Paris-Cité, Paris, France

${ }^{18}$ Rheumatology Unit, Department of Medicine, University of Verona, Verona, Italy

${ }^{19}$ Rheumatology Unit, S. Anna Hospital and University of Ferrara, Ferrara, Italy

${ }^{20}$ Department of Rheumatology, Gaetano Pini Institute, Milan, Italy

${ }^{21}$ Department of Rheumatology, Paul Sabatier University, Toulouse, France

${ }^{22}$ Departments of Medicine and Community Health Sciences, University of Calgary, Calgary, Alberta, Canada

${ }^{23}$ Johns Hopkins University, Baltimore, Maryland, USA

${ }^{24}$ Department of Rheumatology, Ghent University, Ghent, Belgium

${ }^{25}$ Department of Medicine, Cambridge University, Cambridge, United Kingdom

${ }^{26}$ Diakonhjemmet Hospital, Oslo, Norway

${ }^{27}$ CaRE Arthritis LTD, Edmonton, Alberta, Canada

${ }^{28}$ CaRE Arthritis LTD, University of Alberta, Edmonton, Alberta, Canada

Acknowledgements The authors sincerely thank the staff and all patients of the RA BIODAM study.

Contributors All authors made contributions to conception and/or implementation of the study, were involved in reviewing and revising the manuscript and gave final approval to the version to be published.

Funding BIODAM was financially supported by an unrestricted grant from AbbVie. AS is supported by a doctoral grant from 'Fundação para a Ciência e Tecnologia' (SFRH/BD/108246/2015).
Competing interests SR: Received research grants and/or consultancy fees from AbbVie, Eli Lilly, MSD, Novartis and Sanofi. RL: Received consulting fees from AbbVie, BMS, Celgene, Eli-Lilly, Galapagos, Gilead, Glaxo-Smith-Kline, Janssen, Merck, Novartis, Pfizer, Roche, UCB and is Director of Rheumatology Consultancy BV. DvdH: Received consulting fees from AbbVie, Amgen, Astellas, AstraZeneca, BMS, Boehringer Ingelheim, Celgene, Daiichi, Eli-Lilly, Galapagos, Gilead, GlaxoSmith-Kline, Janssen, Merck, Novartis, Pfizer, Regeneron, Roche, Sanofi, Takeda, UCB Pharma and is Director of Imaging Rheumatology BV. AS: Received speaking fees from Novartis. OFG: Received research grants and/or consultancy fees from AbbVie, Eli Lilly, Celgene, Novartis, UCB, Pfizer, Amgen and Janssen. MØ: Received research support, consultancy fees and/or speaker fees form Abbvie, BMS, BoehringerIngelheim, Celgene, Eli-Lilly, Hospira, Janssen, Merck, Novartis, Novo, Orion, Pfizer, Regeneron, Roche, Sandoz, Sanofi and UCB. OE: Received grants and personal fees from Pfizer, Abbvie, Roche, Janssen, Novartis and Lilly. JCT: Received research grants and/or consulting fees from AbbVie, Amgen, Celgene, Centocor, Lilly, Medexux/ Medac, Merck, Novartis, Pfizer, Sandoz and Sanofi. ML: Received advisory board honoraria from AbbVie, Actelion, Boehringer Ingelheim, BMS, Janssen, Novartis, Pfizer, Sanofi, Roche and an unrestricted educational grant from AbbVie. GF: Received research grants and/or consultancy fees from Novartis, Roche, BMS, Pfizer, AbbVie, Lilly, Janssen, MSD, Boehringer-Ingelheim and UCB. MB: Received research grants from AbbVie Co.KG, Roche, Novartis and Pfizer; received speaking fees from Actelion, AbbVie Co.KG, BMS, Celgene, Gilead, Janssen, Lilly, MSD, Novartis, Sanofi Genzyme, Roche, Pfizer and UCB. GB: Received consulting fees from Eli Lilly, Janssen, Novartis and Pfizer; received speaker fees from BMS, Merck and Pfizer. Industry support for investigator-initiated research initiatives from Abbvie, Amgen, BMS, Celgene, Eli Lilly, Jannsen, Merck, Pfizer, Roche and UCB. BC: Received consulting fees from AbbVie, BMS, Eli-Lilly, Gilead, Janssen, Merck, Novartis, Pfizer, Roche-Chugai, Sanofi and UCB. TS: Received consulting fees from Lilly, Novartis, Pfizer and Sanofi; received research financial support from Pfizer and Lilly. AS: Received research grants and/or consultancy fees from AbbVie, BMS, Chugai, Eli Lilly, MSD, Nordic pharma, Novartis, Pfizer, Sanofi and UCB. MD: Received research grants and/or honorarium fees for his participation at advisory boards and/or symposium organised by Pfizer, AbbVie, Lilly, Novartis, BMS, Roche, UCB and Merck. MR: Received advisory board honoraria, consultancy fees and/or speaker fees from Abiogen, Amgen, Abbvie, BMS, Celgene, Eli-Lilly, MSD, Novartis, Pfizer, Sanofi, Sandoz and UCB. MG: Received advisory board honoraria, consultancy fees and/or speaker fees from Abbvie, Alfa-Sigma, BMS, Celgene, MSD, Novartis, Pfizer, Roche and Sanofi. LS: Received speaker fees from Amgen, Eli Lilly, Abbvie, Roche and BMS. AGC: Received consultancy fees from BMS, Chugai, Janssen, Lilly, MSD, Novartis, Pfizer, Roche, Sanofi, UCB, AbbVie, Celgene and Nordic Pharma; received research grants from Pfizer, UCB, AbbVie and Celgene. CB: Received advisory board honoraria from Gilead, Pfizer, Novartis, Eli Lily, Roche and Amgen; speaking fees from UCB, BMS and Abbvie. COB III: Consultant to Abbvie, BMS, Gilead, Lilly, Pfizer, Genentech/Roche and Sanofi/Regeneron. Grant support from BMS and Janssen. PPT: Currently an employee of Kintai Therapeutics, Cambridge MA. Kintai Therapeutics has not been involved in this work. HBH: Received fees for speaking and/or consulting from AbbVie, BMS, Pfizer, UCB, Roche, MSD and Novartis. WPM: Received consulting fees from AbbVie, Boehringer Ingelheim, Celgene, Eli-Lilly, Galapagos, Janssen, Novartis, Pfizer and UCB Pharma and is Chief Medical Officer of CARE Arthritis Limited.

Patient consent for publication Not required.

Ethics approval The study received ethical approval from the local ethics committees.

Provenance and peer review Not commissioned; externally peer reviewed.

Data availability statement Data may be obtained from a third party and are not publicly available.

\section{ORCID iDs}

Sofia Ramiro http://orcid.org/0000-0002-8899-9087

Désirée van der Heijde http://orcid.org/0000-0002-5781-158X

Alexandre Sepriano http://orcid.org/0000-0003-1954-0229

Gilles Boire http://orcid.org/0000-0003-2481-5821

Alain Saraux http://orcid.org/0000-0002-8454-7067

Maurizio Rossini http://orcid.org/0000-0001-9692-2293

Clifton 0 Bingham http://orcid.org/0000-0002-4752-5029

Paul P Tak http://orcid.org/0000-0002-3532-5409

Walter P Maksymowych http://orcid.org/0000-0002-1291-1755

\section{REFERENCES}

1 Kievit W, Fransen J, de Waal Malefijt MC, et al. Treatment changes and improved outcomes in RA: an overview of a large inception cohort from 1989 to 2009 Rheumatology 2013;52:1500-8.

2 Felson DT, Smolen JS, Wells G, et al. American College of Rheumatology/European League against rheumatism provisional definition of remission in rheumatoid arthritis for clinical trials. Ann Rheum Dis 2011;70:404-13. 
3 Smolen JS, Landewé R, Bijlsma J, et al. EULAR recommendations for the management of rheumatoid arthritis with synthetic and biological disease-modifying antirheumatic drugs: 2016 update. Ann Rheum Dis 2017;76:960-77.

4 van Tuyl LHD, Felson DT, Wells G, et al. Evidence for predictive validity of remission on long-term outcome in rheumatoid arthritis: a systematic review. Arthritis Care Res 2010;62:108-17.

5 Schoels M, Knevel R, Aletaha D, et al. Evidence for treating rheumatoid arthritis to target: results of a systematic literature search. Ann Rheum Dis 2010;69:638-43.

6 Schipper LG, van Hulst LTC, Grol R, et al. Meta-Analysis of tight control strategies in rheumatoid arthritis: protocolized treatment has additional value with respect to the clinical outcome. Rheumatology 2010;49:2154-64.

7 Grigor C, Capell H, Stirling A, et al. Effect of a treatment strategy of tight control for rheumatoid arthritis (the TICORA study): a single-blind randomised controlled trial. The Lancet 2004;364:263-9.

8 Verstappen SMM, Jacobs JWG, van der Veen MJ, et al. Intensive treatment with methotrexate in early rheumatoid arthritis: aiming for remission. computer assisted management in early rheumatoid arthritis (camera, an open-label strategy trial). Ann Rheum Dis 2007;66:1443-9.

9 Goekoop-Ruiterman YPM, De Vries-Bouwstra JK, Allaart CF, et al. Clinical and radiographic outcomes of four different treatment strategies in patients with early rheumatoid arthritis (the best study): a randomized, controlled trial. Arthritis Rheum 2005:52:3381-90.

10 Vermeer M, Kuper $\mathrm{HH}$, Hoekstra M, et al. Implementation of a treat-to-target strategy in very early rheumatoid arthritis: results of the Dutch rheumatoid arthritis monitoring remission induction cohort study. Arthritis Rheum 2011;63:2865-72.

11 Schipper LG, Vermeer M, Kuper HH, et al. A tight control treatment strategy aiming for remission in early rheumatoid arthritis is more effective than usual care treatment in daily clinical practice: a study of two cohorts in the Dutch rheumatoid arthritis monitoring registry. Ann Rheum Dis 2012;71:845-50.

12 Brinkmann GH, Norvang V, Norli ES, et al. Treat to target strategy in early rheumatoid arthritis versus routine care - A comparative clinical practice study. Seminars in arthritis and rheumatism 2018.

13 Maksymowych WP, FitzGerald O, Østergaard M, et al. The International RA BIODAM cohort for validation of soluble biomarkers in rheumatoid arthritis: cohort description. J Rheumatol. In Press 2019:jrheum. 190302.

14 Aletaha D, Neogi T, Silman AJ, et al. 2010 rheumatoid arthritis classification criteria: an American College of Rheumatology/European League against rheumatism collaborative initiative. Ann Rheum Dis 2010;69:1580-8.

15 van der Heijde DM, van 't Hof MA, van Riel PL, et al. Judging disease activity in clinical practice in rheumatoid arthritis: first step in the development of a disease activity score. Ann Rheum Dis 1990;49:916-20.

16 Prevoo MLL, van Gestel AM, van T Hof MA, et al. Remission in a prospective study of patients with rheumatoid arthritis. American rheumatism association preliminary remission criteria in relation to the disease activity score. Rheumatology 1996;35:1101-5.
17 Prevoo ML, van 't Hof MA, Kuper HH, et al. Modified disease activity scores that include twenty-eight-joint counts. development and validation in a prospective longitudinal study of patients with rheumatoid arthritis. Arthritis Rheum 1995:38:44-8.

18 Fransen J, Creemers MC, Van Riel PL. Remission in rheumatoid arthritis: agreement of the disease activity score (DAS28) with the Ara preliminary remission criteria. Rheumatology 2004;43:1252-5.

19 Aletaha D, Smolen J. The simplified disease activity index (SDAI) and the clinical disease activity index (CDAI): a review of their usefulness and validity in rheumatoid arthritis. Clin Exp Rheumatol 2005;23:S100-8.

20 Aletaha D, Ward MM, Machold KP, et al. Remission and active disease in rheumatoid arthritis: defining criteria for disease activity states. Arthritis Rheum 2005;52:2625-36

21 van Gestel AM, Prevoo ML, van 't Hof MA, et al. Development and validation of the European League against rheumatism response criteria for rheumatoid arthritis. Comparison with the preliminary American College of rheumatology and the world health Organization/International League against rheumatism criteria. Arthritis Rheum 1996;39:34-40.

22 Sepriano A, Ramiro S, FitzGerald O, et al. Adherence to Treat-to-Target management in rheumatoid arthritis and associated factors: data from the International RA BIODAM cohort. J Rheumatol 2019:jrheum.190303.

23 Markusse IM, Dirven L, Han KH, et al. Evaluating adherence to a Treat-to-Target protocol in recent-onset rheumatoid arthritis: reasons for compliance and hesitation. Arthritis Care Res 2016;68:446-53.

24 Pincus T, Segurado OG. Most visits of most patients with rheumatoid arthritis to most rheumatologists do not include a formal quantitative joint count. Ann Rheum Dis 2006;65:820-2.

25 Yazici Y, Bergman M, Pincus T. Time to score quantitative rheumatoid arthritis measures: 28-Joint count, disease activity score, health assessment questionnaire (HAQ), multidimensional HAQ (MDHAQ), and routine assessment of patient index data (rapid) scores. J Rheumatol 2008;35:603-9.

26 Smolen JS, Breedveld FC, Burmester GR, et al. Treating rheumatoid arthritis to target: 2014 update of the recommendations of an international Task force. Ann Rheum Dis 2016;75:3-15.

27 Solomon DH, Losina E, Lu B, et al. Implementation of Treat-to-Target in rheumatoid arthritis through a learning collaborative: results of a randomized controlled trial. Arthritis Rheumatol 2017:69:1374-80.

28 Gvozdenović E, Allaart CF, van der Heijde D, et al. When rheumatologists report that they agree with a guideline, does this mean that they practise the Guideline in clinical practice? results of the International recommendation implementation study (iris). RMD Open 2016;2:e000221.

29 Landewé RBM. Overdiagnosis and overtreatment in rheumatology: a little caution is in order. Ann Rheum Dis 2018;77:1394-96

30 Bergstra SA, Olivas 0, Akdemir G, et al. Further treatment intensification in undifferentiated and rheumatoid arthritis patients already in low disease activity has limited benefit towards physical functioning. Arthritis Res Ther 2017;19:220. 